\title{
The influence of maternal iron overload on mature rat offspring
}

\author{
JAMES W. KOCHEVAR, JAMES R. MARTIN, BEATRICE D. APPLEBY, \\ J. BRUCE OVERMIER, ROBERT O. FISCH, and WILLIAM KRIVIT \\ University of Minnesota, Minneapolis, Minnesota 55455
}

\begin{abstract}
To determine whether maternal iron overload influences growth and behavior of mature offspring, 10 experimental and 6 control rat offspring were compared on several physical and behavioral dimensions. The experimental offspring did not differ reliably from control offspring in amount of brain iron, proportional weights of organs, number of stress-induced stomach ulcers, general activity level, or performance of a food-rewarded instrumental response. The whole-body weights of experimental offspring were lower than those of controls. Offspring of mothers treated with iron demonstrated greater emotional reactivity than controls, as indexed by persistence of conditioned suppression. These data indicate a need for further investigation of potential toxicity associated with maternal iron overload.
\end{abstract}

To reduce the incidence of iron deficiency anemia in the female and infant populations, it has been suggested that certain foods be fortified with substantially increased levels of iron; in addition, pregnant women in the United States are routinely advised to take iron supplements (Brown, 1972; Committee on Iron Deficiency, AMA, 1968; Finch \& Monsen, 1972). However, Crosby (1970) and Krikker (1972) have issued warnings concerning the possible dangers of dietary iron fortification for the general population, as very little is known about possible deleterious effects of long-term exposure to an iron-rich diet for millions of people who do not require supplemental iron. A frequently cited example of such are the Bantu of South Africa, whose iron-rich diet is a factor probably related to the occurrence of excessive iron deposits within males of the population (Bothwell \& Isaacson, 1962) and, in extreme cases, may result in cirrhosis of the liver, diabetes, and heart failure (see also: Bothwell \& Charlton, 1970; Finch \& Finch, 1955). Equally important, behavioral, personality, and emotional disturbances have been observed in patients suffering from excess-iron-storage diseases (Finch \& Finch, 1955).

This investigation was supported in part by a grant from NIMH (MH-1 3558) to J. B. Overmier, by grants to the University of Minnesota, Center for Research in Human Learning, from the National Science Foundation (GB-35703X), the National Institute of Child Health and Human Development (HD-01136), and the Graduate School of the University of Minnesota, and in part by Public Health Service Grants CA11996 and CA08832 to William Krivit. The authors wish to thank Amos S. Deinard, Linda J. Disch, David Gray, and Mark Jensen for their cooperation and assistance in performing the iron assays of the brains, Raymond Collier for his statistical advice, and Lee J. Levitan for his comments. Reprint requests to: J. B. Overmeir, Department of Psychology, Elliott Hall, University of Minnesota, Minneapolis, Minnesota 55455.
Normally, a homeostatic mechanism involving the mucosal cells of the intestine serves to regulate the quantity of iron absorbed within the body. However, Bothwell and Charlton (1970) suggest that the mechanism may be subject to alteration by the presence of excessive amounts of available iron. Fisch, Deinard, Disch, and Krivit (1975) examined the potential hazards of iron overload with respect to iron transfer from mother to offspring but found no evidence of direct transfer of excess iron from mother to offspring as measured by total body iron at birth. It is possible, however, that even though the offspring of iron-treated females showed no iron overload, as measured by wholebody analysis, the offspring may have had altered iron deposits in select organs; this is because it has been found that depressed levels of nonhaem iron and ferritin iron in brains of rats persisted following short-term exposure to an iron-deficient diet even though iron content of other organs recovered to control levels (Dallman, Siimes, \& Manies, 1975). This suggests that the mechanism for iron absorption in the brain may be differentially affected by manipulations of available iron; the effects might even be cumulative with age of rats, possibly revealing either behavioral or physiological abnormalities (or both) not seen earlier.

Sontag (1941) has noted that abnormal fetal environments can have significant later effects. Thus, by examining the behavior of mature offspring and, later, their individual body organs, it may be possible to detect any indirect effects of maternal iron overload upon development of the offspring.

The present study, then, is a follow-up of the Fisch et al. (1975) study and is characterized as a preliminary attempt to determine whether maternal iron overload may influence the behavior of mature offspring or the iron content and weights of the offspring's selected body organs. 


\section{METHOD}

\section{Subjects}

Sixteen male Sprague-Dawley rats derived from 16 litters, approximately 135 days of age at the outset, served in the present study; 10 were experimental while 6 were control subjects. Experimental animals were offspring of mothers who received intramuscular injections of Imferon (single-dose vials, dextran) iron each week while they were 6 to 12 weeks of age; a total of $240 \mathrm{mg} / \mathrm{kg}$ distributed over 6 weeks was administered (see Fisch et al., 1975, for full details of the experimental treatments). One week after the last injection was given, the females were allowed to mate. No injections were given during pregnancy; however, Fisch et al. (1975) found these mothers maintained the iron overload through parturition. Control animals were offspring of mothers who had not been treated with iron.

During the experiment, three subjects suffered a respiratory illness which required treatment with antibiotics; two were control animals and one was experimental.

\section{Weight and Feeding}

Systematic daily records of each animal's weight were maintained. For the first 38 days of the experiment, the offspring were given 24-h free access to Purina Rat Chow. At the end of this period, subjects were reduced to $80 \%$ of their free-feeding weight and maintained there throughout the behavioral testing portion of the experiment. Two months prior to the time the animals were sacrificed, they were again placed on the freefeeding schedule.

\section{Experimental Manipulations}

To determine whether the prenatal exposure to excessive amounts of iron had major behavioral consequences, the following behaviors were assessed: general activity, learning of a motor response to obtain food, and conditionability and persistence of an emotional reaction. Except for the three ill rats, all subjects received the same treatment on the same day. To prevent differential handling or observation of subjects, the experimenters had no knowledge of which subjects were experimentals and which were controls. Standard electromechanical programming and recording equipment was used.

Activity. Throughout the experiment, prior to obtaining daily weights, each subject was placed in a stabilimeter for $45 \mathrm{~min}$. Later, on days on which conditioning treatments were given, activity measures were taken in the 45 -min period just prior to placement in the operant chamber.

The stabilimeter was a cylindrical cage $30 \mathrm{~cm}$ in diam $\mathrm{x}$ $60 \mathrm{~cm}$ high. The center of the bottom of the cage was attached to a stand by a flexible fulcrum arrangement that allowed the cage to tilt as the subject's weight shifted. This tilting action operated one of four microswitches spaced equally around the base of the cage. The activity measure consisted of total number of closures of the four microswitches during a 45-min period.

Appetitive training. Instrumental training of a leverpress response was initiated 24 days after subjects reached their $80 \%$ deprivation weights. Subjects were given one 45 -min training session per day in a Coulbourn Instruments operant conditioning chamber. The chamber measured $29.5 \times 23.0 \times 28.5 \mathrm{~cm}$ high. The response lever was located on an end wall, $8.0 \mathrm{~cm}$ left of center and $11.0 \mathrm{~cm}$ above the grid floor of the chamber. The food pellet tray was located in the center of the same wall, as was a small houselight and a $4.5-\mathrm{kHz}$ Sonalert which produced the auditory stimuli when appropriate. The operant chamber was within a sound-attenuating chest equipped with a ventilation fan which produced a constant background noise of $80 \mathrm{~dB}$ (re: $20 \mu \mathrm{N} / \mathrm{m}^{2}$ ). Standard $45-\mathrm{mg}$ food pellets were used as reinforcers.

After two daily 45 -min sessions of adaptation to the chamber, one training session on each of the following was given: (1) magazine training, (2) continuous reinforcement for leverpressing, (3) reinforcement on a variable-interval (VI) 30-sec schedule,
(4) on a VI 1-min schedule, and (5) a VI 1.5-min schedule. The animals then received 25 days of training on a VI 2 -min schedule of reinforcement.

Conditioning and extinction of an emotional response. The conditioned suppression procedure was used in the present study to determine whether the experimental and control groups differed in the acquisition or extinction of emotional reactions.

Following appetitive training, habituation to the CS began. During the next 1245 -min sessions, while the subjects continued to earn food on the VI 2-min schedule, a tone CS was presented for $3 \mathrm{~min}$, beginning $10 \mathrm{~min}$ and again at $33 \mathrm{~min}$ into the session. This habituation to the CS insured that it produced no reliable response suppression prior to actual emotional conditioning and should slow acquisition so that group differences might be apparent. Onset of the CS increased the $80-\mathrm{dB}$ background sound level within the chamber by approximately $5 \mathrm{~dB}$.

The next 10 sessions constituted conditioning of the emotional response. The CS was presented for $3 \mathrm{~min}$ as before, but a $.5-\sec 1.0-\mathrm{mA}$ scrambled electric shock US was administered to subjects' feet coincidental with CS termination. The US was delivered by a Grason-Stadler Model E1064GS generator through the grid floor of the operant chamber.

Extinction of the emotional response followed its conditioning. For 10 sessions, the CS was presented twice per session, but the aversive US was no longer presented.

Postmortem physical examination. After completion of behavioral testing, subjects were anesthetized, sacrificed, and thoroughly perfused with saline. Wet weights of the whole brain, liver, spleen, right kidney, and adrenals were obtained. Each stomach was examined to determine the extent of any ulceration that might have resulted during experimental stresses. Iron concentrations of whole brains were determined by atomic absorption spectrophotometry. Details of this procedure were similar to those described by Fisch et al. (1975). Because one experimental subject died after all behavioral studies had been completed but prior to scheduled postmortem examination, its organs were unavailable at the time of examination.

\section{RESULTS}

The criterion for statistical significance was $p<.05$ in all of the following analyses.

\section{Body Weights}

The weights of the animals at 135 and 170 days of age correspond to that portion of the experiment when activity measures were obtained prior to the onset of the restricted feeding schedule. For the experimental offspring these weights were 442 and $446 \mathrm{~g}$, respectively, while for the controls the corresponding weights were 476 and $480 \mathrm{~g}$. The weights at 330 days of age correspond to a point just prior to sacrifice when the rats had been off the restricted feeding schedule for about 2 months: At this time the experimental offspring weighed $438 \mathrm{~g}$, while the controls weighed $462 \mathrm{~g}$. A groups by days analysis of variance for groups with unequal numbers of subjects (ANOVAN) confirmed the observation that, as adults, offspring of mothers treated with iron weighed less than control offspring [groups $\mathrm{F}(1,14)=6.86, \mathrm{p}<.05]$.

It was observed that when the animals were placed on the restricted feeding schedule, there was no difference in the rate at which the two groups reached their criterion $80 \%$ deprivation weight. 


\section{Activity}

Beginning with the last 10 days of free feeding and continuing through extinction of the conditioned emotional response, individual daily activity scores for each group were averaged into successive 5-day blocks. Although the control animals tended to show more activity than the offspring of iron-treated mothers, the intersubject variability was large and a groups by treatments by blocks of days ANOVAN indicated no statistically significant effects attributable to the prenatal exposure to excess iron.

\section{Instrumental Response Rate}

Response rate was determined by counting number of responses occurring within a 3 -min period beginning at a point $30 \mathrm{~min}$ into every training session; this time interval did not overlap with any CS or US presentation during those sessions in which these stimuli were presented. The response rate measure was obained not only during appetitive training, but also during habituation to the CS, conditioning of the emotional response, and extinction of the emotional response. Within each experimental condition, response rates were averaged over five consecutive blocks of 2 days. A groups by treatments by blocks of days ANOVAN revealed that the offspring of mothers treated with iron did not differ from controls overall or within any of the experimental treatments.

\section{Conditioning and Extinction of an Emotional Response}

To assess the degree of relative change in response rate produced by presentation of the signal for shock during acquisition and extinction of the conditioned emotional response, a suppression ratio was calculated for every CS presentation. The formula used to compute the suppression ratios was $b /(a+b)$, where $b$ is the number of leverpresses emitted in the presence of the CS and $a$ is the number of leverpresses emitted in the 3-min period just prior to CS onset (Annau \& Kamin, 1961). To provide a realistic baseline against which suppression ratio changes could be compared, pseudosuppression ratios were also computed using the same formula as that used in the computation of suppression ratios; however, the $a$ and $b$ values used to determine pseudo-suppression ratios were obtained from two adjacent 3-min periods, both of which preceded CS onset by at least $3 \mathrm{~min}$.

Mean real suppression ratios and pseudo-suppression ratios obtained during emotional conditioning and extinction for the two groups are presented in Figure 1. During acquisition of the conditioned emotional response, suppression ratios decreased over days of conditioning, indicating increasing emotional response; pseudo-suppression ratios remained relatively constant. A three-way ANOVAN (groups by pseudo- vs real suppression ratio by days) revealed that both groups acquired the emotional reaction to the CS during the 10 sessions in which the CS was paired with shock [days $\mathrm{F}(4,56)=4.17, \mathrm{p}<.01$; pseudo- vs real suppres- sion ratio $F(1,14)=6.16, p<.05$; days by pseudovs real suppression ratio $F(4,56)=24.67, p<.01]$. Although the experimental group tended to show more suppression during conditioning than the control group, this difference was not reliable [groups $F(1,14)=1.71$ ] .

The suppression ratios for both groups began to recover during extinction of the conditioned emotional response. The pseudo-suppression ratios were more variable during extinction than during conditioning, but they continued to fluctuate around a value of .50 , indicating no suppression. More importantly, the figure suggests that the experimental groups showed more persistent conditioned suppression than controls. A three-way ANOVAN revealed that both groups' suppression to the CS began to diminish during extinction of the emotional reaction [days $F(4,56)=6.08$, $\mathrm{p}<.01]$, but the extent of recovery from suppression during the CS was not complete [days by pseudo- vs real suppression ratio $\mathrm{F}(4,56)=.21$, pseudo- vs real suppression ratio $\mathrm{F}(1,14)=283.17, \mathrm{p}<.01]$. Of special importance is the finding that the animals who were prenatally exposed to excessive iron continued to show a greater degree of response suppression to the CS than did control animals [groups by pseudor vs real suppression ratio $F(1,14)=16.79, p<.01]$.

\section{Postmortem Physical Examination}

To assess whether prenatal exposure to excess iron had differential effects upon the weights of individual organs, it was necessary to partial out the previously determined differences in whole-body weights between the groups. Proportional (organ weight/body weight) organ weights were computed for each subject for each organ. Those were in turn transformed into withinorgans range-corrected scores [ organ $_{x}$ weight subject $_{n}$ - minimum $\operatorname{organ}_{x}$ weight $) /\left(\right.$ maximum $\operatorname{organ}_{x}$ weight

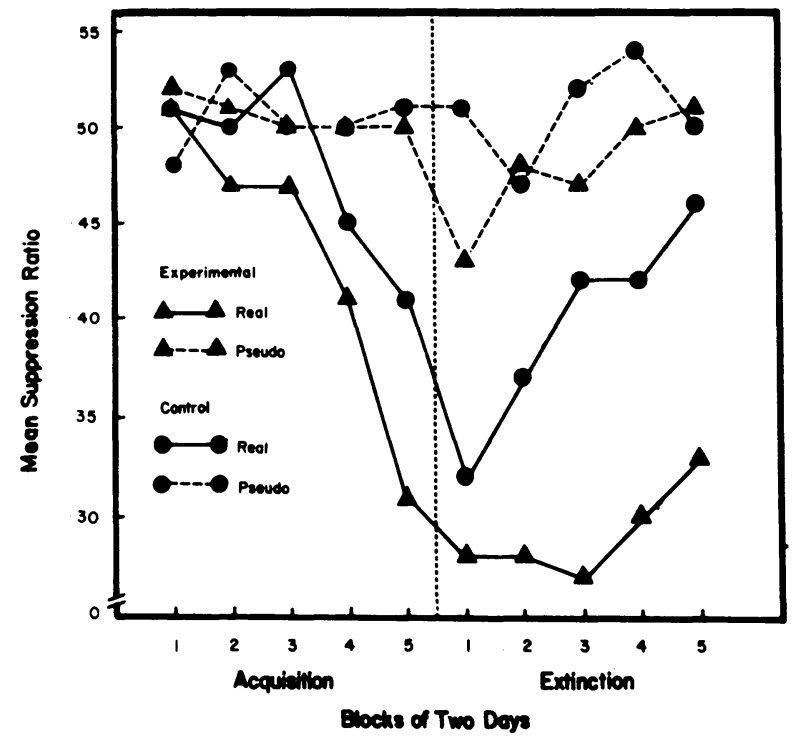

Figure 1. Acquisition and extinction of conditioned emotion response as indexed by mean suppression ratios for the offspring of iron overloaded mothers (EXPERIMENTAL) and for the offspring of normal mothers (CONTROL). 
- minimum organ $_{x}$ weight)] to facilitate comparison in a groups by organ ANOVAN. This analysis revealed no reliable difference in the proportional organ weights between the experimental and control groups for brain, liver, spleen, kidney, or adrenals.

Total number of ulcers in both glandular and nonglandular portions of the animals' stomachs were counted. The offspring of iron-treated mothers had an average of 1.89 ulcers, while the control animals had an average of 2.17 ulcers, a difference which was not significant $[\mathrm{t}(13)=.23]$.

Analysis of the iron content of the rats' brains revealed that experimental subjects had an average of $77.6 \mu \mathrm{g}$ of iron/g brain tissue, while control subjects had an average of $73.6 \mu \mathrm{g}$ of iron/g brain tissue. Although the experimental offspring had a greater amount of iron in their brains than did control offspring, the difference in iron content was not statistically reliable $[\mathrm{t}(13)=1.17]$.

\section{DISCUSSION}

The present results indicated limited physiological and behavioral effects of prenatal exposure to excessive iron upon mature rat offspring. For example, the mean mature body weight of offspring of iron-treated mothers was less than that of control animals. However, experimental animals did not differ significantly from control offspring in amount of brain iron, proportional weights of body organs, or extent of stomach ulceration resulting from stress of experimentation.

Behaviorally, during extinction of the conditioned emotional response, offspring of iron-treated mothers demonstrated a greater conditioned emotional response than did control animals. However, the experimental and control offspring were similar in general activity level and performance of an appetitively maintained instrumental response.

It is possible that the greater degree of conditioned suppression reflects greater emotional reactivity. In this regard, it is interesting to note that emotional disturbances, depression, suicidal tendencies, or psychoneurosis have been reported in human patients suffering from idiopathic hemochromatosis, which is associated with exposure to excessive amounts of iron (Finch \& Finch, 1955). Thus, if exposure to excessive amounts of iron per se has behavioral consequences, as these anecdotes suggest, the consequences or our experimental manipulation might reasonably be expected to be manifest primarily in the emotional components of behavior.

It has been shown that growth and later behavior of rat offspring can be influenced by pre- and postnatal maternal emotionality (Denenberg, Ottinger, \& Stephens, 1962). To the extent that maternal behavior can affect the behavior of mature offspring, it is important to note that the design of the present experiment permitted two major alternative explanations of the results. The first is a direct influence of maternal iron status upon the iron metabolism of the offspring. Second, the excessive iron could have affected the mothers' behavior (emotionality?), which in turn modulated the offsprings' behavior. Although this second possibility represents an indirect influence, it would still reflect iron-related effects of no small importance, especially when this animal model is applied to the issue of iron supplementation for human mothers.

Finally, we wish to note that the mothers of control offspring, while equally of ten handled, were not given sham injections. Various forms of stimulation administered to mothers during their infancy or their pregnancy may influence the behavior of offspring, although the observation of a behavior change and its direction is not consistent (Ader \& Conklin, 1963; Hockman, 1961; Whiting, 1967). But, important for the present experiment is the recognition that the injections of the mothers, here, were prior to pregnancy. There are no demonstrations of effects of adult prepregnancy handling upon the behavior of the offspring. Thus, the effects observed here are most probably attributable to the iron status of the mothers during pregnancy.

In conclusion, it was found that excess amounts of iron in the mother may result in rat offspring who weigh less and are more emotionally reactive at maturity than control offspring. In view of current attempts to increase the amount of iron supplementation in the foods we eat, the present results argue for the initiation of further research designed to examine the physiological and psychological consequences of maternal iron overload.

\section{REFERENCES}

ADER, R., \& ConkiIN, P. M. Handling of pregnant rats: Effects on emotionality of their offspring. Science, 1963, 142, 411-412.

American Medical Association Council on Foods and Nutrition, Committee on IRON DEficiency. Iron deficiency in the United States. Journal of the American Medical Association, 1968, 203, 407-412.

Annau, Z., \& Kamin, L. J. The conditioned emotional response as a function of the intensity of the US. Journal of Comparative and Physiological Psychology, 1961, 54, 428-432.

Bothwell, T. H., \& Charlton, R. W. Absorption of iron. Annual Review of Medicine, 1970, 21, 145-156.

Bothwell, T. H., \& IsaAcson, C. Siderosis in the Bantu: Incidence in males and females. British Medical Journal, $1962,1,522-524$.

Brown, E. B. Irony. British Journal of Haematology, 1972, 23, 97-100.

CRosBy, W. H. Iron enrichment, one's food, another's poison. Archives of Internal Medicine, 1970, 126, 911-913.

Dallman, P. R., Sirmes, M. A., \& Manies, E. C. Brain iron: Persistent deficiency following short-term iron deprivation in the young rat. British Journal of Haematology, 1975, 31, 209-215.

Denenberg, V. H., Ottinger, D. R., \& Stephens, M. W. Effects of maternal factors upon growth and behavior of the rat. Child Development, 1962, 33, 65-71.

Finch, C. A., \& Monsen, E. R. Iron nutrition and the fortification of food with iron. Journal of the American Medical Association, 1972, 219, 1462-1465.

Finch, S. C., \& Finch, C. A. Idiopathic hemochromatosis, an iron storage disease. Medicine, 1955, 34, 381-421.

Fisch, R. O., Deinard, A. S., Disch, L. J., \& Krivit, W. Potential toxicity of iron overload in successive generations of rats. American Journal of Clinical Nutrition, 1975, 28, 136-139.

Hockman, C. H. Prenatal maternal stress in the rat: Its effects on emotional behavior in the offspring. Journal of Comparative and Physiological Psychology, 1961, 54, 679-684.

KRIKKER, M. A. Excessive fortification of flour with iron. New England Journal of Medicine, 1972, 286, 162-163.

Sontag, L. W. The significance of fetal environmental differences. American Journal of Obstetrics and Gynecology, 1941, 42, 996-1003.

Whiting, J. W. M. Analysis of infant stimulation. In D. C. Glass (Ed.), Neurophysiology and emotion. New York: Rockefeller University Press \& Russell Sage Foundation, 1967.

(Received for publication February 28, 1977.) 